\title{
Bat diversity of the Serranía de San Lucas (Bolívar and Antioquia), northern Colombia
}

\author{
Sergio Solari ${ }^{1}{ }^{2 *}$, Daisy Gómez-Ruiz ${ }^{1,3}{ }^{3}$, Edilson Patiño-Castillo ${ }^{1}$, Tomás Villada-Cadavid ${ }^{1}$, y Carolina López M. ${ }^{1}$ \\ ${ }^{1}$ Grupo Mastozoología, Universidad de Antioquia. Dirección de correspondencia: Calle 70 No. 52-21, Medellín, AA 1226. \\ Antioquia, Colombia. Email: sergio.solari@udea.edu.co (SS), dalejandra.gomez@hotmail.es (DG-R), edipaca1989@hotmail.com \\ (EP-C), tomasir012@gmail.com (TV-C), carolin.0880@gmail.com (CLM). \\ ${ }^{2}$ Instituto de Biología de la Universidad de Antioquia. Dirección de correspondencia: Calle 70 No. 52-21, Medellín, AA 1226, \\ Antioquia, Colombia. \\ ${ }^{3}$ Facultad de Medicina Veterinaria, Corporación Universitaria Remington. Dirección de correspondencia: Calle 51 No. 51-27, \\ Medellín, Antioquia, Colombia. \\ * Corresponding author
}

Serranía de San Lucas is an isolated mountain ridge in the Northern Andes, reaching above 2,500 masl, located between the Central and the Eastern Cordilleras in northeast Colombia, including parts of Bolívar and Antioquia departments. Due to its particular location and difficult access, there are few studies on its biological diversity. In order to clarify the geographical affinities of its bat fauna, we conducted a survey at four representative localities of this mountain range. Between 2015 and 2017, we conducted four assessments to characterize the bat species richness in San Lucas; these localities represent the dominant habitats in the area, including lowland, premontane forests, and swamps. We used three to six mist nets per night, between 17:30 and 23:00 h and identified species in the field, collecting specimens to corroborate and document the diversity in each location. Species accumulation curves and non-parametric estimators assessed efficiency of sampling; in addition, Jaccard's similarity index was used to assess beta diversity. Each species was assigned to a trophic guild for ecological analyses and comparisons of the assemblages. We recorded 47 species from six families, with Phyllostomidae being the most diverse (35 species). Although species richness at a local scale varied between 14 and 23 species, the heterogeneity among them shows a particular richness for San Lucas as a whole. A notable geographic record was Mimon cozumelae, which extends its known distribution in northern South America, while other four species are confirmed for the Magdalena Valley, reinforcing a trans-Andean influence. Our records reinforce a biogeographical connection with the region of Chocó-Nechí-Magdalena, thus contributing to validation of the perception that San Lucas shows greater affinity to the Central Cordillera than to the Eastern one. It is evident that several groups (i. e., insectivorous) have not been fully sampled, and that new methods (diurnal roost searches and acoustical sampling) and longer survey efforts might reveal more additions to this list. Our study reveals some peculiarities in the diversity of bats of Serranía de San Lucas, including species typical of inter-Andean valleys and suggesting an influence of the trans-Andean zone, resulting in major similarities to assemblages from northwestern Colombia and South America, but with a specific composition that is indicative of the good state of conservation of the habitats in the study area and its relevance as a future, and necessary, protected area for the country.

La Serranía de San Lucas constituye un ramal montañoso aislado de los Andes del Norte, alcanzando más de 2,500 msnm, entre las cordilleras Central y Oriental, en el nororiente de Colombia, incluyendo parte de los departamentos Bolívar y Antioquia. Debido a su ubicación particular y difícil acceso, son pocos los estudios sobre su diversidad biológica. A fin de clarificar las afinidades geográficas de su quiropterofauna, se propuso un muestreo en localidades representativas de esta serranía. Entre 2015 y 2017, se realizaron cuatro evaluaciones a fin de caracterizar la riqueza de murciélagos de la serranía; estas localidades representan hábitats dominantes en la zona, incluyendo bosques de tierras bajas, bosques premontanos y ciénagas. Se utilizaron de tres a seis redes de niebla por noche, entre las 17:30 y 23:00 h. Se identificaron las especies en campo, recolectando ejemplares para corroborar y documentar la diversidad en cada localidad. Se realizaron curvas de acumulación de especies y estimadores no paramétricos para evaluar la eficiencia del muestreo, adicionalmente se calculó el índice de similitud de Jaccard para evaluar la diversidad beta. Cada especie fue asignada a un gremio trófico para los análisis ecológicos y comparaciones del ensamblaje. Registramos 47 especies pertenecientes a seis familias, siendo Phyllostomidae la más diversa (35 especies). Aunque la riqueza de especies a escala local varió entre 14 y 23 especies, la heterogeneidad entre ellas muestra una riqueza particular para la serranía de San Lucas. Registros geográficos notables incluyen a Mimon cozumelae que extiende su distribución en el norte de Sudamérica; mientras cuatro especies son confirmadas para el valle del Magdalena, reforzando una influencia transandina. Nuestros registros refuerzan una conexión biogeográfica con la región del Chocó-Nechí-Magdalena, lo cual contribuye a validar la percepción que San Lucas muestra mayor afinidad a la cordillera Central que a la Oriental. Se evidencia que muchos grupos (i. e., insectívoros) no han sido exhaustivamente muestreados y es posible que nuevas metodologías (búsqueda de refugios diurnos y muestreo acústico) y mayores esfuerzos en otras localidades revelen más adiciones a la lista. Nuestro estudio revela ciertas particularidades en la diversidad de murciélagos de esta serranía, incluyendo especies típicas de valles interandinos y sugiriendo una influencia de la zona Transandina, con mayores similitudes respecto a ensamblajes del noroccidente de Colombia y Sudamérica, aunque su composición específica también indica el buen estado de conservación de los hábitats situados en la zona de los muestreos y su relevancia como una futura, y necesaria, área protegida en el país.

Keywords: assemblages; Chiroptera; distribution; northern Andes; species richness.

(C 2020 Asociación Mexicana de Mastozoología, www.mastozoologiamexicana.org 


\section{Introducción}

Durante los últimos años, se han encaminado esfuerzos notables para actualizar el conocimiento de los mamíferos en Colombia; una reciente actualización concluyó con el registro de 492 especies silvestres (Solari et al. 2013). A partir de revisiones taxonómicas o adiciones de nuevas localidades de distribución para varias especies neotropicales, este número se incrementó a 500 para el año 2014 (Ramírez-Chaves y Suárez-Castro 2014). Dos años después, se incrementó a 518 (Ramírez-Chaves et al. 2016), y el estimado más reciente incluye 528 especies (Ramírez-Chaves et al. 2018). Estos cambios reflejan la complejidad del estudio sistemático y taxonómico de los mamíferos en el país, incluyendo la constante revisión de colecciones y el estudio de nuevas áreas, a pesar de ser comparativamente mejor conocidos que otros vertebrados.

Un dato destacable en estos estudios ha sido la verificación que el grupo más diverso es el de los murciélagos (orden Chiroptera) con alrededor de 209 especies, seguido por los roedores (orden Rodentia) con alrededor de 132 especies. Al igual que la generalidad de los mamíferos, los murciélagos no se encuentran homogéneamente distribuidos en el país, debido a la gran variedad de hábitats y climas presentes, esperándose una mayor diversidad de especies en las zonas tropicales (Kattan et al. 2004; Mantilla-Meluk et al. 2009; Solari et al. 2013; Mantilla-Meluk et al. 2014). Los murciélagos son importantes elementos de los ecosistemas de bosque tropical; ellos influyen en la estructura, composición y dinámica de estos sistemas a través de procesos como la dispersión de semillas, polinización, e impactos sobre poblaciones de insectos (Kunz et al. 2011), y sirven como presas potenciales para diferentes depredadores como algunos mamíferos, búhos y serpientes (Gillette y Kimbourgh 1970). Por ello, se consideran indicadores de disturbios ambientales causados tanto por el hombre o por cambios climáticos drásticos (Jones et al. 2009), en adición a los importantes servicios ecosistémicos mencionados anteriormente.

Una de las zonas de alta montaña más interesantes de Colombia, virtualmente inexplorada, es la Serranía de San Lucas, cubierta en su mayor parte por selvas húmedas frecuentemente nubladas (Castaño-Uribe et al. 1999; Salaman et al. 2002). Se trata de una serranía topológicamente compleja y accidentada, lo cual ha permitido sugerir que presenta una biota bastante endémica, probablemente de afinidad andina (Hernández-Camacho et al. 1992), pero de la cual no se tienen datos empíricos que soporten dicha idea (Salaman et al. 2002). Este potencial endemismo también podría extenderse hacia los mamíferos, incluyendo los murciélagos.

La adecuada caracterización de la biodiversidad de una región como la Serranía de San Lucas, requiere la obtención de listados taxonómicos precisos y actualizados. Para esta región solamente existen estimados preliminares de la diversidad de mamíferos y murciélagos (Castaño-Uribe et al. 1999). Listas previas de murciélagos en zonas próximas a la Serranía San Lucas (pero no al interior), como Hershkovitz
(1949) para dos localidades del departamento de Bolívar, incluye solo nueve especies, mientras que un estudio en la vereda La Cruz, municipio de Remedios (J. Campuzano, com. pers.) incluye resultados de varios muestreos realizados durante un año en un fragmento de bosque húmedo. Este último estudio resultó en una lista que incluye 19 especies.

Debido a estas imprecisiones en cuanto a datos sobre la diversidad, el propósito de nuestra investigación en la Serranía de San Lucas incluye la descripción y caracterización de la diversidad de murciélagos en esta región para mejorar nuestro entendimiento de sus afinidades faunísticas. Debido a su posición aislada respecto a la Cordillera Central, entender sus afinidades permitirá evaluar algunas interrogantes biogeográficas y ecológicas previamente planteadas respecto a los límites de la región denominada Chocó-Nechí-Magdalena (Hernández-Camacho et al. 1992).

\section{Metodología}

Área de estudio. La Serranía de San Lucas constituye el extremo norte de la Cordillera Central, ocupando la región sur del Departamento de Bolívar y el extremo oriental del Departamento de Antioquia, con una extensión aproximada de $200 \mathrm{~km}$ (norte-sur), alcanzando alturas de 2,700 m. Esta serranía está aislada de la Cordillera Central por el Río Nechí, que inunda una planicie en su extremo nordeste; así, sobre la isolínea de los 1,000 m, la serranía se encuentra separada por más de $75 \mathrm{~km}$ de la Cordillera Central, y por casi $65 \mathrm{~km}$ de la Cordillera Oriental, por el valle del Magdalena Medio colombiano (Figura 1). Desde el punto de vista biológico, se ha considerado como un enigma debido a que la inestabilidad política y social, acentuada por un prolongado conflicto armado, ha impedido el acceso a la zona, y cuando esto ha sido posible solo se ha logrado estudiar el límite inferior, por debajo de 1,500 m, y con esfuerzos limitados de muestreo, mayormente por avistamientos (Salaman et al. 2002). Las localidades visitadas se encuentran dentro del zonobioma del bosque húmedo tropical (sensu Hernández-Camacho et al. 1992), incluyendo mosaicos de paisaje con una matriz paisajística de bosques primarios, bosques secundarios en avanzado estado de regeneración, bosques riparios, pastizales, rastrojos, riveras de quebradas y ciénagas. Además, algunos de estos sitios están dedicados a la conservación a partir de iniciativas comunitarias como los encontrados en las localidades Ojos Claros y Cerro 1800.

Captura de animales. Entre abril de 2015 y febrero de 2017 se realizaron cuatro expediciones en diferentes localidades y épocas climáticas en las estribaciones de la Serranía de San Lucas (Tabla 1). Para la captura de murciélagos, se usaron de tres a seis redes por noche (6 o $12 \mathrm{~m}$ de largo), que estuvieron activas, en promedio, desde las 17:30 hasta las 23:00 horas. Las redes fueron revisadas cada 30 minutos o menos, según la actividad de los murciélagos en cada sitio y en cada noche. Se seleccionaron, primariamente, áreas naturales con poca intervención humana, pero también buscando incluir diferentes hábitats representativos. 


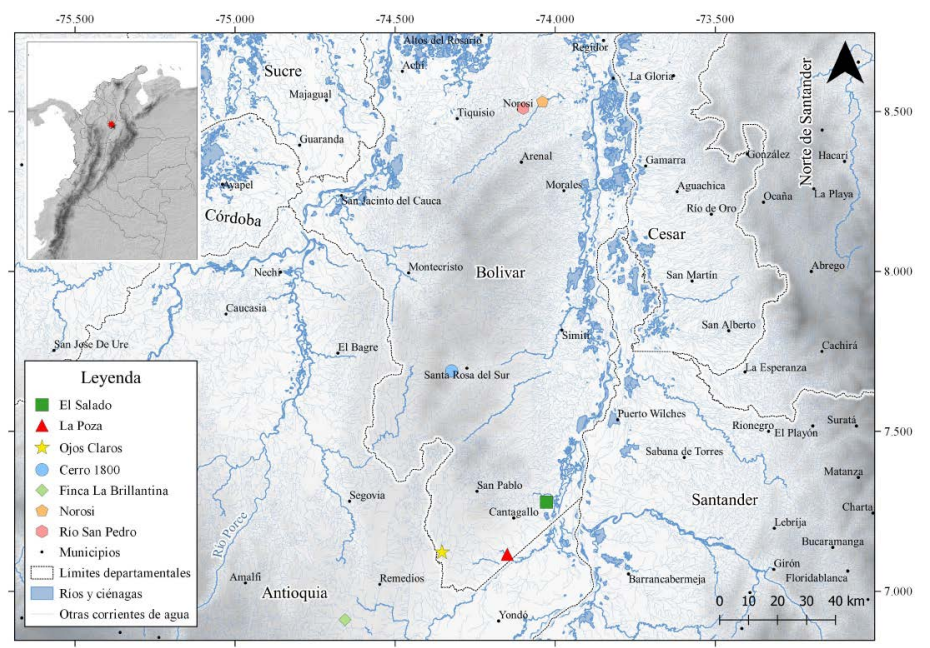

Figura 1. Ubicación de las cuatro localidades de muestreo en la Serranía de San Lucas en la región sur del Departamento de Bolívar y el extremo oriental del Departamento de Antioquia. Se incluye las localidades referenciadas en el texto: Norosí y Río San Pedro (norte de Bolívar; Hershkovitz 1949) y Hacienda La Brillantina, Remedios (oriente de Antioquia; J. Campuzano, no publ.).

Los murciélagos capturados fueron medidos y examinados (medidas externas, peso, estado reproductivo, condición general y presencia de ectoparásitos) para facilitar su determinación taxonómica. Esta determinación se realizó mediante el uso de las claves pertinentes (Gardner 2008; Díazetal. 2016), hasta el nivel taxonómico que éstas lo permitieron (género o especie). A fin de documentar adecuadamente esta diversidad, algunos ejemplares se colectaron y prepararon según los procedimientos sugeridos por Sikes et al. (2016), siendo preservados como cuerpos completos y carcasas en alcohol, así como pieles disecadas, cráneos y esqueletos. De cada individuo colectado se tomó una muestra de tejido hepático para futuros estudios de identificación genética, también se colectaron ectoparásitos que se almacenaron en etanol al 96 $\%$. Para las especies más comunes, los individuos ya identificados se liberaron en la misma cobertura donde fueron capturados. Todo el material colectado fue depositado en la Colección Teriológica de la Universidad de Antioquia (CTUA).

Tabla 1. Localidades incluidas en el muestreo, fechas y época de cada expedición, y esfuerzos de muestreo empleados en cada sitio. Las fechas de cada salida incluyen tanto las noches de muestreo efectivo como el acceso a cada localidad.

\begin{tabular}{|c|c|c|c|c|}
\hline Localidad & Coordenadas & $\begin{array}{c}\text { Elevación } \\
\text { (msnm) }\end{array}$ & $\begin{array}{c}\text { Fechas y } \\
\text { época }\end{array}$ & $\begin{array}{c}\text { Esfuerzo } \\
\text { (m²-hrs- } \\
\text { noche) }\end{array}$ \\
\hline \multicolumn{5}{|c|}{ Departamento Antioquia } \\
\hline $\begin{array}{l}\text { Municipio de Remedios, } \\
\text { Vereda Ojos Claros }\end{array}$ & $\begin{array}{r}74^{\circ} 21^{\prime} 36.9^{\prime \prime} \mathrm{W} \\
7^{\circ} 06^{\prime} 54.1^{\prime \prime} \mathrm{N}\end{array}$ & $290-509$ & $\begin{array}{c}14 \text { abr }-09 \\
\text { may de } 2015 \\
\quad(\text { seca })\end{array}$ & 7,632 \\
\hline \multicolumn{5}{|c|}{ Departamento Bolívar } \\
\hline $\begin{array}{l}\text { Municipio Santa Rosa } \\
\text { del Sur, Vereda San Juan, } \\
\text { Cerro } 1800\end{array}$ & $\begin{array}{r}74^{\circ} 19^{\prime} 20.8^{\prime \prime} \mathrm{W} \\
7^{\circ} 41^{\prime} 00.5^{\prime \prime} \mathrm{N}\end{array}$ & $1003-1225$ & $\begin{array}{l}22-29 \text { jul de } \\
2015 \text { (seca) }\end{array}$ & 1,290 \\
\hline $\begin{array}{l}\text { Municipio Cantagallo, } \\
\text { Vereda EI Salado }\end{array}$ & $\begin{array}{l}74^{\circ} 16^{\prime} 53.5^{\prime \prime} \mathrm{W} \\
07^{\circ} 16^{\prime} 54.6^{\prime \prime} \mathrm{N}\end{array}$ & $91-134$ & $\begin{array}{l}16-31 \text { oct de } \\
2016 \text { (lluvias) }\end{array}$ & 1,266 \\
\hline $\begin{array}{l}\text { Municipio Cantagallo, } \\
\text { Vereda La Poza }\end{array}$ & $\begin{array}{l}74^{\circ} 9^{\prime} 5.2^{\prime \prime} \mathrm{W} \\
7^{\circ} 6^{\prime} 26.1^{\prime \prime} \mathrm{N}\end{array}$ & $85-159$ & $\begin{array}{c}10-27 \text { feb de } \\
2017 \text { (seca) }\end{array}$ & 1,836 \\
\hline
\end{tabular}

Análisis de datos. Para evaluar la representatividad del muestreo construimos curvas de rarefacción de especies y calculamos los estimadores no paramétricos Chao1 (abundancia) y Chao2 (incidencia) empleando el programa EstimateS 9.1.0 (Colwell 2013). Generamos dichas curvas basadas en individuos con el fin de hacer comparaciones entre los sitios de muestreo (Colwell y Coddington 1994), debido a las diferencias en los esfuerzos de muestreo empleados. Adicionalmente, se realizó un análisis de diversidad beta calculando la similitud entre las cuatro localidades (Moreno 2001), para discutir las diferencias entre la composición de especies de estos sitios; un clúster jerárquico se realizó empleando un análisis calculado a partir del índice de similitud de Jaccard, en el programa PAST 3 (Hammer et al. 2001).

Como una primera aproximación al estudio del ensamblaje de murciélagos en la Serranía de San Lucas, se empleó la matriz de nichos tróficos y tallas desarrollada por $\mathrm{McNab}$ (1971) y empleada informativamente para caracterizar ensamblajes similares en Trinidad, Panamá, Costa Rica, Brasil, y la Guyana (Willig 1986; Lim y Engstrom 2001). Este sistema predice que solo una especie común ocupará cada celda de esta matriz de doble entrada, lo que abre la posibilidad de expandir la discusión a partir de las celdas donde más de una especie ocurre. Las categorías de nicho trófico siguen aquellas sugeridas por LaVal y Fitch (1977), y ampliadas por Patterson et al. (1996) y Lim y Engstrom (2001); las categorías de peso siguen la sugerencia por Lim y Engstrom (2001). A pesar de algunas limitaciones en este diseño (ver Discusión), consideramos que el aporte informativo es valioso para esta caracterización del ensamblaje.

Adicionalmente, se realizó un análisis del rol funcional que cumplen las especies de murciélagos, siguiendo a Soriano (2000). Esto se logra caracterizando los nichos y roles tróficos de cada especie, para obtener los equivalentes tróficos (ET) de cada gremio y estimar el porcentaje de aporte de cada gremio al ensamblaje total. Solo en el caso de Trinycteris nicefori se cambió la categorización sugerida por Soriano (2000) a una más acorde a lo que se conoce de su historia natural (Gardner 2008). Una ventaja de este análisis es que también permite la comparación de los resultados entre sitios de estudio, aumentando la perspectiva de uso de estos hallazgos.

\section{Resultados}

Luego de más de 50 noches efectivas de muestreo, se completó un esfuerzo de 12,024 $\mathrm{m}^{2}$-horas-noche (Tabla 1), registrando un total de 590 individuos, e identificando 47 especies de murciélagos (Tabla 2) pertenecientes a seis familias y 33 géneros. En la localidad Ojos Claros registramos 23 especies (148 individuos), en el Cerro 1800 registramos 14 especies (79 individuos), en El Salado, 22 especies ( 283 individuos) y en La Poza, 21 especies ( 80 individuos). Algunos registros notables incluyen siete especies de insectívoros de follaje (Gardnerycteris keenani, Lampronycteris brachyotis, Lophostoma silvicola, Micronycteris hirsuta, M. megalotis, Mimon cozumelae, y Tonatia saurophila), y doce especies 
Tabla 2. Especies de murciélagos y número de individuos capturados en cada una de las localidades (ver Figura 5).

\begin{tabular}{|c|c|c|c|c|c|}
\hline Familia & Especie & $\begin{array}{l}\text { Ojos } \\
\text { Claros }\end{array}$ & $\begin{array}{l}\text { Cerro } \\
1800\end{array}$ & El Salado & La Poza \\
\hline \multirow[t]{5}{*}{ Emballonuridae } & Centronycteris centralis & & & & 1 \\
\hline & Rhynchonycteris naso & & & 2 & \\
\hline & Saccopteryx bilineata & 1 & & 1 & \\
\hline & Saccopteryx canescens & & & 1 & \\
\hline & Saccopteryx leptura & & & & 1 \\
\hline Mormoopidae & Pteronotus parnellii & 1 & & & 2 \\
\hline \multirow[t]{2}{*}{ Noctilionidae } & Noctilio albiventris & & & 8 & 2 \\
\hline & Noctilio leporinus & & & 2 & \\
\hline \multirow[t]{35}{*}{ Phyllostomidae } & Desmodus rotundus & 2 & & 2 & \\
\hline & Chrotopterus auritus & & & 1 & \\
\hline & Gardnerycteris keenani & 1 & 1 & & 1 \\
\hline & Lophostoma silvicola & 1 & & & 3 \\
\hline & Micronycteris hirsuta & 1 & & & \\
\hline & Micronycteris megalotis & & & 2 & \\
\hline & Mimon cozumelae & 1 & & & \\
\hline & Phyllostomus discolor & & & 1 & 2 \\
\hline & Phyllostomus hastatus & & & 1 & 1 \\
\hline & Tonatia saurophila & & 2 & & 1 \\
\hline & Trachops cirrhosus & 7 & & & \\
\hline & Trinycteris nicefori & & & & 1 \\
\hline & Lampronycteris brachyotis & 1 & & & \\
\hline & Anoura cultrata & & 1 & & \\
\hline & Glossophaga soricina & & & 4 & \\
\hline & Hsunycteris thomasi & 4 & & & \\
\hline & Lionycteris spurrelli & 2 & & & \\
\hline & Lonchophylla robusta & & 2 & & \\
\hline & Carollia brevicauda & 51 & 30 & & \\
\hline & Carollia castanea & 9 & 5 & 48 & 16 \\
\hline & Carollia perspicillata & 34 & 6 & 130 & 15 \\
\hline & Artibeus lituratus & 4 & 15 & 19 & \\
\hline & Artibeus phaeotis & 5 & 2 & 3 & 1 \\
\hline & Artibeus planirostris & 4 & & 15 & 8 \\
\hline & Artibeus ravus & & & 10 & \\
\hline & Chiroderma salvini & & 1 & & \\
\hline & Chiroderma trinitatum & & & & 1 \\
\hline & Chiroderma villosum & 2 & & 1 & \\
\hline & Platyrrhinus dorsalis & & 3 & & \\
\hline & Platyrrhinus helleri & 2 & & 9 & 3 \\
\hline & Sturnira bakeri & 1 & 8 & 3 & 4 \\
\hline & Uroderma convexum & & & 18 & 6 \\
\hline & Vampyressa thyone & 1 & 2 & & 1 \\
\hline & Vampyriscus nymphaea & 10 & & & \\
\hline & Vampyrodes major & 2 & & & \\
\hline \multirow[t]{2}{*}{ Molossidae } & Molossops temminckii & & & & 1 \\
\hline & Molossus molossus & & & 2 & 9 \\
\hline \multirow[t]{2}{*}{ Vespertilionidae } & Myotis caucensis & 1 & & & \\
\hline & Myotis riparius & & 1 & & \\
\hline Total de individuos & & 148 & 79 & 283 & 80 \\
\hline Total especies & & 23 & 14 & 22 & 21 \\
\hline
\end{tabular}

representando las familias insectívoras Emballonuridae (5), Molossidae (2), Vespertilionidae (2), Noctilionidae (2) y Mormoopidae (1).
Al analizar la representatividad del muestreo por localidad, se observa un rápido incremento en la acumulación de especies a medida que aumentan los individuos capturados, pero ninguna de las curvas alcanza una asíntota (Apéndice 1). Con base en el esfuerzo realizado (Tabla 1), los estimadores de riqueza varían entre localidades (Apéndice 2) mostrando que la representatividad del muestreo puede estar por debajo del 70 \% (Ojos Claros, 62.7\%, La Poza, 65.4 $\%$ ) o por encima del $85 \%$ (El Salado, $88 \%$, Cerro 1800, 92.2 $\%)$. En conjunto con las curvas de rarefacción, esto indica que, en localidades como Ojos Claros y la Poza, los ensamblajes estarían compuestos por muchas más especies de las registradas en el presente estudio.

El análisis de la composición de especies muestra una baja similitud entre los ensamblajes en cada localidad. Solo tres de las especies (Sturnira bakeri, Artibeus phaeotis, y CaroIlia castanea) fueron comunes a todas las localidades, mientras que se registraron entre cinco y nueve especies únicas por localidad (El Salado: 5 especies; La Poza: 6; Ojos Claros: 8; y Cerro 1800: 9; Tabla 2). Respecto a las especies compartidas entre localidades, se encuentra que las localidades de tierras bajas y hábitats asociados a ciénagas (La Poza y El Salado) presentan ensamblajes más similares entre sí, compartiendo el $34 \%$ de las especies. Por otro lado, la localidad más disímil con respecto a las otras es Cerro 1800, compartiendo solo $16 \%$ de las especies con El Salado, y $25 \%$, con La Poza. Po otro lado, Cerro 1800 y Ojos Claros comparten $23 \%$ de sus especies, con ocho especies en común (Tabla 2). El clúster jerárquico (Figura 2) muestra estas similitudes.

Solamente 23 de las 48 celdas posibles en la matriz de nichos tróficos/pesos para el ensamblaje de la Serranía de San Lucas estuvieron ocupadas (48\%; Apéndice 3). Solo una especie de cada uno, carnívoros/piscívoros, omnívoros y frugívoros nómadas, ocuparon la clase de mayor peso; pero, mientras que los frugívoros nómadas muestran la mayor variación en tallas (cinco celdas, solo ausentes de la celda < $6 \mathrm{~g}$ ), los dos grupos de insectívoros, aéreos y de follaje, y los nectarívoros ocupan las categorías de menor peso, aunque solo los insectívoros aéreos ocupan la celda de menor peso $(<6 \mathrm{~g})$. En cuanto al análisis de roles funcionales, el gremio de frugívoros nómadas representó el mayor aportante, con el $26.8 \%$, seguido por los insectívoros aéreos con $21.3 \%$, y los insectívoros de follaje con $21.1 \%$ (Apéndice 4).

\section{Discusión}

En este primer estudio de la quiropterofauna de la Serranía de San Lucas nuestro esfuerzo estuvo limitado al uso de redes de niebla, con sus correspondientes virtudes y defectos (Simmons y Voss 1998; Kunz y Parsons 2009); aun así, nuestros hallazgos muestran un ensamblaje característico de la complejidad de hábitats muestreados en el área de estudio. Por esta razón, los análisis y comparaciones a continuación se hacen con cautela y deben tomarse como preliminares (Fleming 1986; Willig 1986; Kalko et al. 1996). En este caso, y dada las condiciones particulares de este muestreo, se combinaron los datos de las cuatro localidades en la 


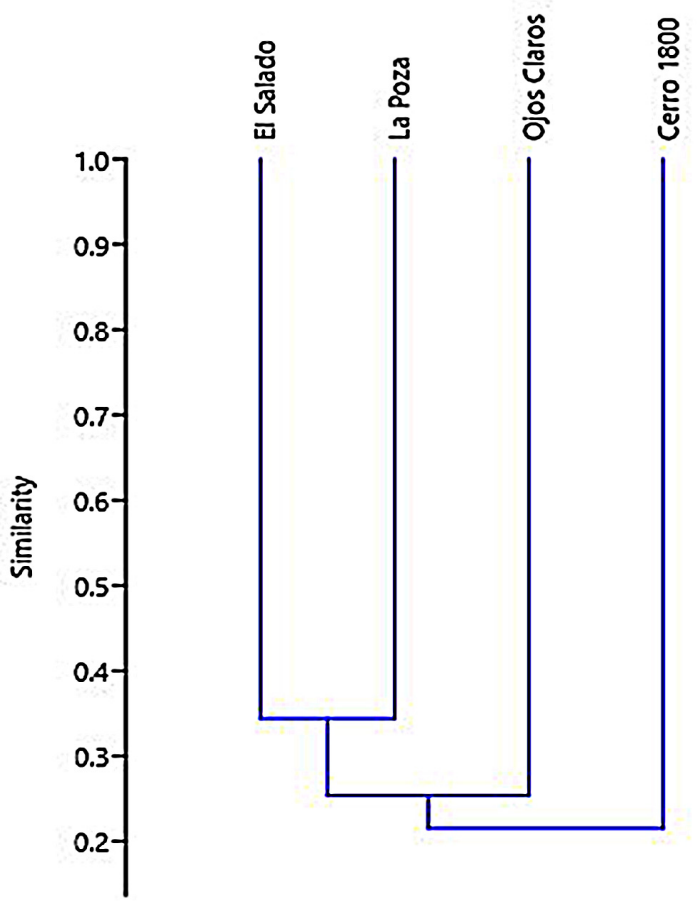

Figura 2. Clúster jerárquico de los cuatro ensamblajes en la Serranía de San Lucas, a partir del índice de Jaccard.

Serranía de San Lucas a fin de comparar cualitativamente la lista resultante (Tabla 2 ) con evaluaciones similares para el valle del Magdalena, Tolima (i. e., Zona I en Bejarano-Bonilla et al. 2007) o Río Claro, en el oriente de Antioquia (Muñoz 1986).

Un primer aspecto a resaltar es que en San Lucas la fauna de murciélagos es más diversa (47 spp.) que la del valle del Magdalena (28 spp.) o Río Claro (27 spp.); sin embargo, esta diferencia no se debe a la presencia de especies endémicas a la serranía, ni a un muestreo más completo de la diversidad regional. Así, algunas especies, como Molossops planirostris o Promops centralis (valle del Magdalena) no fueron registradas por nosotros, ni tampoco Anoura caudifer (Río Claro). Otras especies "faltantes" incluirían a Phylloderma stenops, Vampyrum spectrum (en Remedios, E Antioquia; J. Campuzano, no publ.) y Eumops auripendulus (en Norosí, N Bolívar; Hershkovitz 1949); otras especies potenciales para esta región incluirían a Thyroptera tricolor (Thyropteridae), Lonchorhina aurita y algunos nectarívoros de las subfamilias Glossophaginae y Lonchophyllinae (Phyllostomidae). Esto puede ser resultado de las limitaciones propias de una primera evaluación, que no se evaluaron todos los hábitats de la zona, o que no se emplearon otras técnicas para el registro de especies, incluyendo la búsqueda de refugios diurnos o el muestreo del estrato arbóreo (Simmons y Voss 1998), y el registro de llamadas de ecolocación (Kalko et al. 1996; Zurc et al. 2017). Sin embargo, nuestra lista incluye especies como Vampyrodes major, Vampyriscus nymphaea, y Mimon cozumelae, que además de aportar al conocimiento sobre sus distribuciones geográficas, también sugieren una influencia de las regiones Chocó, Nechí y Magdalena, algo discutido previamente por otros autores (Hernández-Camacho et al. 1992; Martínez-Arias y Solari 2013). Es intere- sante que esta conexión en particular había sido indicada a partir de los estudios de la avifauna en el Cerro 1800 (Salaman et al. 2002).

En cuanto a la composición específica obtenida en el muestreo, llama la atención la captura de algunas especies que, aunque representadas por solamente de uno a dos individuos, dan idea de un ensamblaje particular y confirman el valor del gremio de especies insectívoras como un adecuado indicador del estado de conservación (Jones et al. 2009). La alta diversidad de este grupo se asocia a la existencia de ambientes con poca intervención humana (Fenton et al. 1992; Medellín et al. 2000), debido al rol ecológico ligado a sus dietas. El monitoreo de la diversidad y de las poblaciones de especies de insectívoros aéreos e insectívoros de follaje en asociación con los bosques que habitan puede ser útil para evaluar el efecto de perturbaciones ambientales (Solari et al. 2002; Díaz-Pulido et al. 2015).

Registros notables. Respecto a la distribución de especies, el hallazgo de Mimon cozumelae (Figura 3A) en Ojos Claros representaría el segundo registro de la especie para Colombia y para Sudamérica (Williams y Genoways 2008), siendo el registro previo un ejemplar de Chigorodó (Antioquia; Marinkelle y Cadena 1972; Solari et al. 2013). Es decir, el registro obtenido en la vereda Ojos Claros representa una extensión geográfica de más de $250 \mathrm{~km}$ al este de la distribución conocida. La distribución del género en Colombia (Solari et al. 2013; Hurtado y Pacheco 2014) es bastante restringida con registros correspondientes a $M$. bennettii para Socorré (Bolívar; Gregorin et al. 2008), Serranía de la Macarena (Meta; Cuervo-Díaz et al. 1986) y Serranía de Chiribiquete (Caquetá; Montenegro y Romero-Ruiz 1999).

El ejemplar CTUA 2406 es un individuo juvenil, macho, con osificación incompleta de las epífisis de las falanges y escaso o nulo gasto dental. Sin embargo, muestra todas las características que permiten asignarlo a este género. Aunque la taxonomía del género y la distinción entre especies es compleja (Hoppe y Ditchfield 2016), las medidas cráneo-dentales (Apéndice 5) y las características craneales y dentales (Figura 3B) permiten confirmar la identificación. Por ejemplo, la fosa mesopterigoidea (en forma de V) y la caja craneana poco inflada, y dentalmente, el tamaño relativo del talónido del tercer molar inferior son también característicos de $M$. cozumelae. Igualmente, la amplitud del tabique medio del proceso palatino en la premaxila, corresponde a la estructura ósea ubicada entre los forámenes incisivos, es ancha y muestra un foramen accesorio medio, que de acuerdo con Hurtado y Pacheco (2014) también ayudan a distinguir a esta especie de M. bennettii. La única discordancia presentada por este ejemplar fue la forma de los incisivos superiores, que se adelgaza para formar una punta (como en M. bennettii) en lugar de formar un borde amplio. Consideramos que esto podría deberse a la condición juvenil del ejemplar, con los dientes mostrando poco gasto, o a la variabilidad geográfica indicada por Hoppe y Ditchfield (2016). Además, coincidente con algunas descripciones, este ejemplar muestra la punta de 

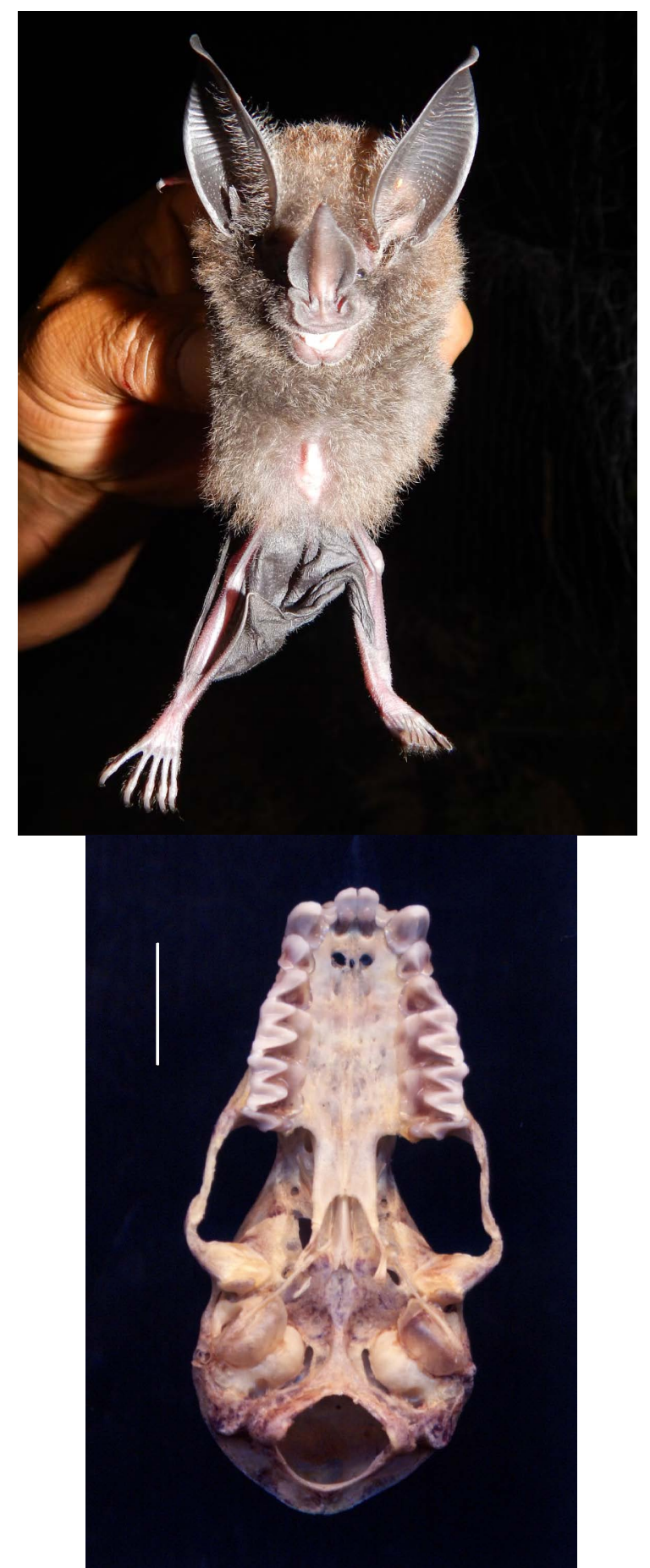

Figura 3. Mimon cozumelae (foto por J. Mosquera), registrado en la localidad Ojos Claros; a la derecha, detalles cráneo-dentales útiles para la identificación de la especie.

las alas de color blanquecino, así como manchas blancas en la base de las orejas (Dalquest 1957; Simmons y Voss 1998).

Otras especies poco comunes de este ensamblaje fueron Pteronotus parnellii, Molossops temminckii, Lampronycteris brachyotis, Vampyriscus nymphaea, y Vampyrodes major. Este hecho no significa que sean poco abundantes (o "raras") en la región, ya que más bien podría deberse a posibles sesgos metodológicos del muestreo. Inclusive, algunos de estos registros aportan al conocimiento de la distribución de las especies, como en el caso de V. nymphaea y $V$. major (Apéndice 6) que constituyen los primeros registros confirmados para el valle del Magdalena (Velazco y
Simmons 2011; Rodríguez-Posada y Ramírez-Chaves 2012). También resultó llamativo el hecho que las cinco especies de embalonúridos fueran registradas en El Salado y La Poza, que constituyen ambientes con presencia de ciénagas y que son estacionalmente inundables (elevaciones por debajo de 200 m), y representativas del Magdalena Medio colombiano. Son igualmente importantes que dos especies nectarívoras, como Lonchophylla robusta y Anoura cultrata (Apéndice 6), solo fueran registradas, con solo un individuo en cada caso, en la localidad de Cerro 1800. Sin duda, esto muestra el escaso conocimiento que hay sobre estos ensamblajes y la necesidad de realizar más estudios sobre el reemplazo altitudinal en cadenas montañosas aisladas (Kattan et al. 2004).

Limitaciones metodológicas del muestreo. Debe resaltarse que estudios de mayor esfuerzo y duración, así como empleando una combinación de otras técnicas (Simmons y Voss 1998) resultan imprescindibles para lograr inventarios más completos. Los resultados indican que el muestreo no alcanzó una adecuada representatividad (> $85 \%$, Apéndice 2) en localidades como Ojos Claros y La Poza, indicando que aún faltan especies por registrar y que la composición regional del ensamblaje puede ser mayor a la esperada. Por otro lado, aunque el menor valor de riqueza específica se dio en Cerro 1800, el estimador de abundancia muestra una representatividad de $92 \%$ (Apéndice 2), a pesar de ser la localidad donde se empleó el menor esfuerzo de muestreo (Tabla 1). Esto puede estar relacionado con los sesgos metodológicos antes señalados, pero también con las diferencias respecto al hábitat y estado de conservación de los sitios muestreados, las cuales aumentan la posibilidad de encontrar nuevas especies con un esfuerzo de muestreo más prolongado, en las mismas localidades o en otras con condiciones de hábitat similares.

Diversidad ecológica del ensamblaje. La descripción ecológica del ensamblaje se hizo mediante dos análisis que, aunque basan su premisa principal en una similar caracterización de los nichos tróficos, muestran diferencias en sus resultados, pero también coinciden en que hay una interesante diversidad de grupos ecológicos. Se ha sugerido que la incorporación de otros parámetros ecológicos (uso del dosel arbóreo, abundancia relativa, dieta) a estas matrices permitiría una evaluación más detallada de la segregación ecológica entre las especies, y un mejor entendimiento de la conformación de estos ensamblajes (Willig 1986).

Una característica particular del análisis de la matriz de nichos tróficos/pesos es que hubo solo una especie "común" (abundante) en las celdas en las categorías de pesos por sobre los $20 \mathrm{~g}$, que corresponde a Artibeus lituratus, lo cual es una diferencia con los resultados mostrados por Lim y Engstrom (2001) para Iwokrama. La forma particular de esta matriz sugiere que hay una distribución "natural" de las especies, con especies pequeñas ( $<20 \mathrm{~g}$ ) caracterizando los gremios insectívoros aéreos y nectarívoros, mientras que las especies de mayor talla (> $37 \mathrm{~g}$ ) caracterizan a los carnívoros/piscívoros y omnívoros. Otro dato de interés es que 12 celdas estuvieron ocupadas por solo una especie, 
lo que sugiere que aún hay "espacio" para otras especies en este ensamblaje, y que el muestreo no ha logrado completar una adecuada representatividad.

Conservación de la Serranía de San Lucas. Nuestros hallazgos resultan remarcables para la conservación de esta región desde el contexto geográfico, ecológico y social, porque comprenden los primeros muestreos dirigidos y sistemáticos de la quiropterofauna de la Serranía de San Lucas, además de incluir la colecta de ejemplares que permiten verificar estas identificaciones para documentar y/o verificar los patrones sugeridos de riqueza y endemismo de especies en esta región tan aislada (Castaño-Uribe et al. 1999; Salaman y Donegan 2001). Dada la escasez de estudios similares, esta información será útil para futuros análisis del estado de conservación, distribución y taxonomía de la fauna asociada a esta serranía. En esa misma línea, estos registros permitirán corroborar la relevancia de especies, ensamblajes y ambientes que merezcan ser parte de programas de conservación, así como estudios sistemáticos precisos y análisis biogeográficos más finos, que una vez se incorporen a los planes de manejo existentes contribuirán a la delimitación precisa de esta zona para su reconocimiento como área protegida en el corto plazo. Al mismo tiempo, nuevos diseños podrían proponerse para el estudio de ciertas especializaciones ecológicas, como en el caso de la dieta, actividad, y selección de refugios por especies de murciélagos. A largo plazo, aspectos puntuales de estos patrones podrían incorporarse a esquemas de monitoreo de las condiciones ambientales que las soportan.

Las particularidades de los registros obtenidos hasta este momento son alentadoras para futuros estudios, pero el estado de amenaza de estos bosques, debido a los avances de la minería informal, extracción maderera no regulada, y la situación de orden público, representan un riesgo cuya solución no puede ser postergada. El reconocimiento de estas características únicas de su biodiversidad, así como de los servicios ecosistémicos que ella presta, son un paso importante en ese sentido. Es de esperar que mayores estudios en esta región ayuden a completar algunos de los vacíos actuales para un mejor entendimiento del ensamblaje de murciélagos y sus roles ecológicos dentro de la Serranía de San Lucas.

\section{Agradecimientos}

Este estudio fue posible gracias a la invitación y el apoyo logístico de la Dirección de Parques Nacionales Naturales de Colombia, Wildlife Conservation Society (WCS), la Asociación Campesina del Valle del río Cimitarra (ACVC) y la Corporación Autónoma Regional del Centro de Antioquia (CORANTIOQUIA). Institucionalmente, el Instituto de Biología, Universidad de Antioquia, facilitó la participación del primer autor, mientras que el Grupo Mastozoología colaboró con los equipos para el trabajo de campo y recepción del material recolectado. Agradecemos a L. Dary-Acevedo, W. Bonell, y J. Mosquera por su apoyo durante el trabajo de campo, y a esta última por compartir algunas fotos de las especies. El trabajo de campo se convirtió en una extraordinaria experiencia de aprendizaje gracias a la activa participación de las comunidades campesinas en Ojos Claros, Santa Rosa del Sur, El Salado y La Poza; su compromiso con la pacificación y la protección de esta zona es una valiosa lección que aporta al componente social de estos resultados. Finalmente, a los evaluadores de una primera versión del manuscrito por las valiosas sugerencias que mejoraron sustancialmente este documento.

\section{Literatura Citada}

Bejarano-Bonilla, D. A., A. Yate-Rivas, y M. H. Bernal-Bautista. 2007. Diversidad y distribución de la fauna de quirópteros en un transecto altitudinal en el departamento del Tolima, Colombia. Caldasia 29:297-308.

Castaño-Uribe, C., C. Gómez-Durán, y A. Hurtado-García. 1999. Sierras y Serranías de Colombia. Banco de Occidente. Bogotá, Colombia.

ColweLl, R. K. 2013. EstimateS: Statistical estimation of species richness and shared species from samples. Version 9.1.0. User's Guide and application published at: http://purl.oclc. org/estimates.

Colwell, R. K., y J. A. Coddington. 1994. Estimating terrestrial biodiversity through extrapolation. Philosophical Transactions of the Royal Society of London. Series B: Biological Sciences 345:101-118.

Cuervo-Díaz, A. J. Hernández-Camacho, y A. Cadena G. 1986. Lista actualizada de los mamíferos de Colombia anotaciones sobre su distribución. Caldasia 15:471-501.

Dalquest, W. W. 1957. American bats of the genus Mimon. Proceedings of the Biological Society of Washington 70:45-47.

Diaz, M. M., S. Solari, L. F. Aguirre, L. Aguiar, y R. M. Barquez. 2016. Clave de identificación de los murciélagos de Sudamérica. PCMA (Programa de Conservación de los Murciélagos de Argentina). Publicación Especial № 2. Tucumán, Argentina.

Díaz-Pulido, A., M. Aguilar-Garavito, J. Pérez-Torres, y S. Solari. 2015. El monitoreo de los mamíferos en los procesos de restauración ecológica. Pp. 163-176 in Monitoreo a procesos de restauración ecológica aplicado a ecosistemas terrestres (Aguilar-Garavito, M., y W. Ramírez, eds.). Instituto de Investigación de Recursos Biológicos Alexander von Humboldt (IAvH). Bogotá, Colombia.

Fenton, M. B., L. Acharya, D. Audet, M. B. C. Hickey, C. Merriman, M. K. Obrist, y D. M. Syme. 1992. Phyllostomid bats (Chiroptera: Phyllostomidae) as indicators of habitat disruption in the Neotropics. Biotropica 24:440-446.

Fleming, T. H. 1986. The structure of Neotropical bat communities: a preliminary analysis. Revista Chilena de Historia Natural 59:135-150.

GaRDNeR, A. L. 2008. Mammals of South America, vol. 1. Marsupials, Xenarthrans, Shrews, and Bats. The University of Chicago Press, Chicago, EE.UU.

Gillette, D. D., y J. D. KıмвouRGH. 1970. Chiropteran mortality. Pp. 262-281 in About bats (Slaughter, B. H., y D. W. Walton, eds.). Dallas Southern Methodist University Press. Dallas, EE.UU. 
Gregorin, R., G. L. Capusso, y V. R. Furtado. 2008. Geographic distribution and morphological variation in Mimon bennettii (Chiroptera, Phyllostomidae). Iheringia 98:404-411.

Hammer, Ø., D. A. T. Harper, y P. D. Ryan. 2001. PAST: Paleontological statistics software package for education and data analysis. Palaeontologia Electronica 4:1-9. http:// palaeo-electronica.org/2001_1/past/issue1_01.htm

Hernández-Camacho, J., A. Hurtado-Guerra, R. Ortiz-Quijano, y T. Walschburger. 1992. Unidades biogeográficas de Colombia. Pp. 105-151 in La diversidad biológica de Iberoamérica. Vol. I (G. Halffter, Ed.). Acta Zoológica Mexicana, Nueva Serie, Vol. Especial. Ciudad de México, México.

Hershkovitz, P. 1949. Mammals of Northern Colombia, preliminary report No. 5: Bats (Chiroptera). Proceedings of the United States National Museum 99:429-454.

Hoppe, J. P. M., y A. D. Ditchfield. 2016. Range extension of Mimon bennettii (Chiroptera: Phyllostomidae) in Brazil with comments on its systematics. Mammalia 80:469-473.

Hurtado, N., y V. Pacheco. 2014. Análisis filogenético del género Mimon Gray, 1847 (Mammalia, Chiroptera, Phyllostomidae) con la descripción de un nuevo género. Therya 5:751-791.

Jones, G., D. S. Jacobs, T. H. Kunz, M. R. Willig, y P. A. Racey. 2009. Carpe noctem: the importance of bats as bioindicators. Endangered Species Research 8:93-115.

Kalko, E. K., C. O. Handley, JR., y D. Handley. 1996. Organization, diversity, and long-term dynamics of a Neotropical bat community. Pp. 503-553 in Long-term studies of vertebrate communities (Cody, M. L. y Smallwood, J. A. eds). Academic Press, Inc. San Diego, EE.UU.

Kattan, G. H., P. Franco, V. Rojas, y G. Morales. 2004. Biological diversification in a complex region: a spatial analysis of faunistic diversity and biogeography of the Andes of Colombia. Journal of Biogeography 31:1829-1839.

Kunz, T. H., y S. Parsons. 2009. Ecological and behavioral methods for the study of bats. segunda edición. The John Hopkins University Press. Baltimore, EE.UU.

Kunz, T. H., E. B. De Torrez, D. Bauer, T. Lobova, y T. H. Fleming. 2011. Ecosystem services provided by bats. Annals of the New York Academy of Sciences 1223:1-38.

LAVAL, R. K., Y H. S. Fitch. 1977. Structure, movements and reproduction in three Costa Rican bat communities. Occasional Papers, Museum of Natural History of the University of Kansas 69:1-28.

Lim, B. K., y M. D. Engstrom. 2001. Bat community structure at Iwokrama Forest, Guyana. Journal of Tropical Ecology 17:647-665.

Mantilla-Meluk, H., A. M. Jiménez-Ortega, y R. J. Baker. 2009. Phyllostomid bats of Colombia: Annotated checklist, distribution, and biogeography. Special Publications, Museum of Texas Tech University 56:1-37.

Mantilla-Meluk, H., H. E. Ramírez-Chaves, A. M. JiménezOrtega, y M. E. Rodríguez-Posada. 2014. Emballonurid bats from Colombia: Annotated checklist, distribution, and biogeography. Therya 5:229-255.

Marinkelle, C. J., y A. Cadena. 1972. Notes on bats new to the fauna of Colombia. Mammalia 36:49-58.

Martínez-Arias, V. M., y S. Soları. 2013. Extensión del área de distribución de Sturnira koopmanhilli McCarthy et al. 2006 en Colombia. Therya 4:617-625.
McNaB, B. K. 1971. The structure of tropical bat faunas. Ecology 52:352-358.

Medellín, R. A., M. Equihua, y M. A. Amin. 2000. Bat diversity and abundance as indicators of disturbance in Neotropical rainforests. Conservation Biology 14:1666-1675.

Moreno, C. E. 2001. Métodos para medir la biodiversidad. M\&T-Manuales y Tesis SEA, vol. 1. Zaragoza, 10.15472/kl1whs.

Montenegro, O. L., y M. Romero-Ruiz. 1999. Murciélagos del sector sur de la serranía de Chiribiquete, Caquetá, Colombia. Revista da Academia Colombiana de Ciencias Exactas, Físicas y Naturales 23:641-649.

Muñoz A., J. 1986. Murciélagos del Parque Natural El Refugio (Antioquia, Colombia. Actualidades Biológicas 15:66-76.

Patterson, B. D., V. Pacheco, y S. Solari. 1996. Distribution of bats along an elevational gradient in the Andes of southeastern Peru. Journal of Zoology 240:637-658.

Ramírez-Chaves, H. E., y A. F. Suárez-Castro. 2014. Adiciones y cambios a la lista de mamíferos de Colombia: 500 especies registradas para el territorio nacional. Mammalogy Notes 1:223-235.

Ramírez-Chaves, H. E., A. F. Suárez-Castro, y J. González-Maya. 2016. Cambios recientes a la lista de los mamíferos de Colombia. Mammalogy Notes 3:1-9.

Ramírez-Chaves, H., A. F. Suárez-Castro, Sociedad Colombiana de Mastozoología, D. Zurc, D. C. Concha-Osbahr, F. Trujillo, E. A. Noguera-Urbano, G. E. Pantoja, M. Rodríguez-Posada, J. F. González-Maya, J. Pérez-Torres, H. Mantilla-Meluk, C. López, A. Velásquez, y D. Zárrate-Charry. 2018. Mamíferos de Colombia. Versión 1.5. Sociedad Colombiana de Mastozoología (Checklist). http://doi.org/10.15472/kl1whs

Rodríguez-Posada, M.E., y H. Ramírez-Chaves. 2012. Distribución, morfología y reproducción del murciélago rayado de orejas amarillas Vampyriscus nymphaea (Mammalia: Chiroptera) en Colombia. Acta Zoológica Mexicana (n. s.) 28:341-352.

Salaman P. G. W., y T. M. Donegan (eds.). 2001. Presenting the first biological assessment of Serranía de San Lucas. Colombian EBA Project Report Series No. 3. Published online by Fundación ProAves, Colombia at www.proaves.org.

Salaman P. G. W., T. M. Donegan, y A. M. Cuervo. 2002. New distributional bird records from Serranía de San Lucas and adjacent Central Cordillera of Colombia. Bulletin of the British Ornithologists Club 122:285-303.

Sikes, R. S., y The Animal Care and Use Committee of the American Society of Mammalogists. 2016. Guidelines of the American Society of Mammalogists for the use of wild mammals in research and education. Journal of Mammalogy 97:663-688.

Simmons, N. B., y R. S. Voss. 1998. The mammals of Paracou, French Guiana: a Neotropical lowland rainforest fauna. Part 1. Bats. Bulletin of the American Museum of Natural History 237:1-219.

Soları, S., J. J. Rodríguez, E. Vivar, y P. M. Velazco. 2002. A framework for assessment and monitoring of small mammals in a lowland tropical forest. Environmental Monitoring and Assessment 76:89-104.

Solari, S., Y. Muñoz-Saba, J. V. Rodríguez-Mahecha, T. R. Defler, H. E. Ramírez-Chaves, y F. Trujillo. 2013. Riqueza, endemismo y conservación de los mamíferos de Colombia. Mastozoología Neotropical 20:301-365. 
Soriano, P. J. 2000. Functional structure of bat communities in tropical rainforests and andean cloud forests. Ecotrópicos 13:1-20.

Velazco, P. M., y N. B. Simmons. 2011. Systematics and taxonomy of great striped-faced bats of the genus Vampyrodes Thomas, 1900 (Chiroptera: Phyllostomidae). American Museum Novitates 3710:1-35.

Willaams, S. L., Y H. H. Genoways. 2008. Subfamily Phyllostominae Gray, 1825. Pp. 255-300, in Mammals of South America. Volume 1. Marsupials, Xenarthrans, Shrews, and Bats (Gardner, A. L., ed.). The University of Chicago Press, Chicago, EE.UU.

WILLIG, M. R. 1986. Bat community structure in South America: a tenacious chimera. Revista Chilena de Historia Natural 59:151-168.

Zurc, D., A. Guillén-Servant, y S. Solari. 2017. Chillidos de ecolocación de murciélagos de la familia Emballonuridae en un bosque Sseco tropical del Caribe Colombiano. Mastozoología Neotropical 24:201-218.

Associated editor: Robert Owen

Submitted: June 23, 2019; Reviewed: July 23, 2019;

Accepted:October 15, 2019; Published on line:December 31, 2019.

\section{APÉNDICES}

Apéndice 1. Curvas de rarefacción de especies de murciélagos en la Serranía de San Lucas por localidades de muestreo. A. Ojos Claros. B. Cerro 1800. C. El Salado. D. La Poza.

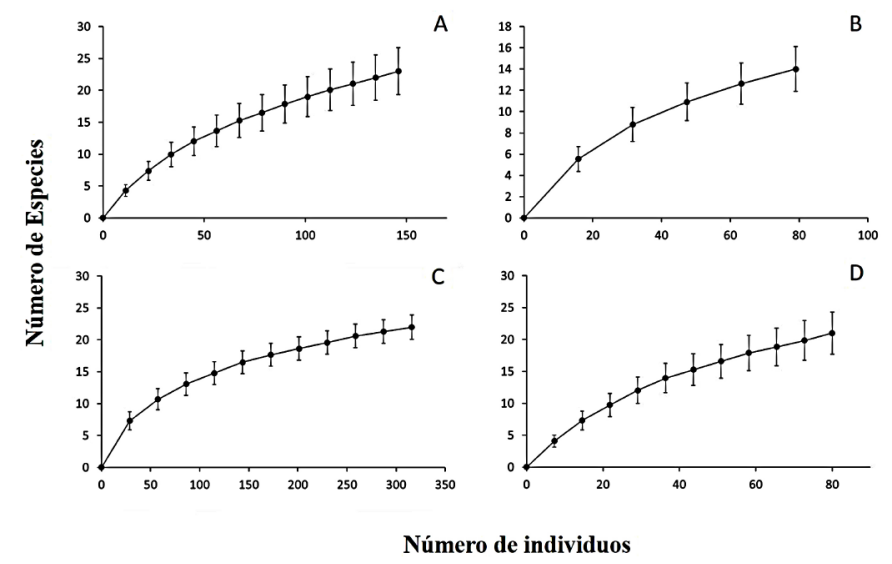

Apéndice 2. Estimadores de riqueza basados en abundancia e incidencia por localidad de muestreo en la Serranía de San Lucas.

\begin{tabular}{lccccc}
\hline Localidad & & $\begin{array}{c}\text { Ojos } \\
\text { Claros }\end{array}$ & $\begin{array}{c}\text { Cerro } \\
\mathbf{1 8 0 0}\end{array}$ & El Salado & La Poza \\
\hline $\begin{array}{l}\text { Riqueza de } \\
\text { especies }\end{array}$ & 23 & 14 & 22 & 21 \\
$\begin{array}{l}\text { Estimador de } \\
\text { Riqueza }\end{array}$ & & & & & \\
Abundancia & Chao1 & $36.66 \pm$ & $15.18 \pm$ & $24.99 \pm$ & $32.11 \pm$ \\
& & 11.11 & 1.82 & 3.41 & 9.45 \\
Incidencia & Chao2 & $43.31 \pm$ & $18.20 \pm$ & $25.18 \pm$ & $36.00 \pm$ \\
& & 16.19 & 4.40 & 3.37 & 11.8 \\
Completitud & & $62.70 \%$ & $92.20 \%$ & $88.00 \%$ & $65.40 \%$ \\
\hline
\end{tabular}

Apéndice 3. Matriz de nicho trófico y tallas para los murciélagos registrados en la Serranía de San Lucas durante el estudio. El número en paréntesis indica el número de especies comunes (o relativamente abundantes) en dicha celda.

\begin{tabular}{|c|c|c|c|c|c|c|c|}
\hline Nicho trófico & $\begin{array}{c}<6 \\
\mathrm{~g}\end{array}$ & $\begin{array}{c}6-10 \\
g\end{array}$ & $\begin{array}{c}11-19 \\
g\end{array}$ & $\begin{array}{c}20-36 \\
\mathrm{~g}\end{array}$ & $\begin{array}{c}37-69 \\
\mathrm{~g}\end{array}$ & $\begin{array}{c}>69 \\
\mathrm{~g}\end{array}$ & Total \\
\hline $\begin{array}{l}\text { Insectívoros } \\
\text { aéreos }\end{array}$ & $\begin{array}{c}6 \\
(1)\end{array}$ & 2 & 1 & 2 & & & 11 \\
\hline $\begin{array}{l}\text { Insectívoros de } \\
\text { follaje }\end{array}$ & & 2 & 2 & 3 & & & 7 \\
\hline Nectarívoros & & $3(1)$ & 2 & & & & 5 \\
\hline $\begin{array}{l}\text { Frugívoros } \\
\text { sedentarios }\end{array}$ & & & $3(2)$ & 1 & & & 4 \\
\hline $\begin{array}{l}\text { Frugívoros } \\
\text { nómadas }\end{array}$ & & 2 & $6(2)$ & 3 & 1 & $1(1)$ & 13 \\
\hline Omnívoros & & & 1 & 1 & & 1 & 3 \\
\hline $\begin{array}{l}\text { Carnívoros/ } \\
\text { Piscívoros }\end{array}$ & & & & 1 & 1 & 1 & 3 \\
\hline Hematófagos & & & & 1 & & & 1 \\
\hline Total especies & 6 & 9 & 15 & 12 & 2 & 3 & 47 \\
\hline
\end{tabular}


Apéndice 4. Equivalentes tróficos y categorías funcionales del ensamblaje de murciélagos (basado en Soriano, 2000). PIS: Piscívoros, CAR: Carnívoros, SAN: Sanguinívoros, GLE: Insectívoros de follaje, INS: Insectívoros aéreos, FRU_N: Frugívoros nómadas, FRU_S: Frugívoros sedentarios, NEC: Nectarívoros.

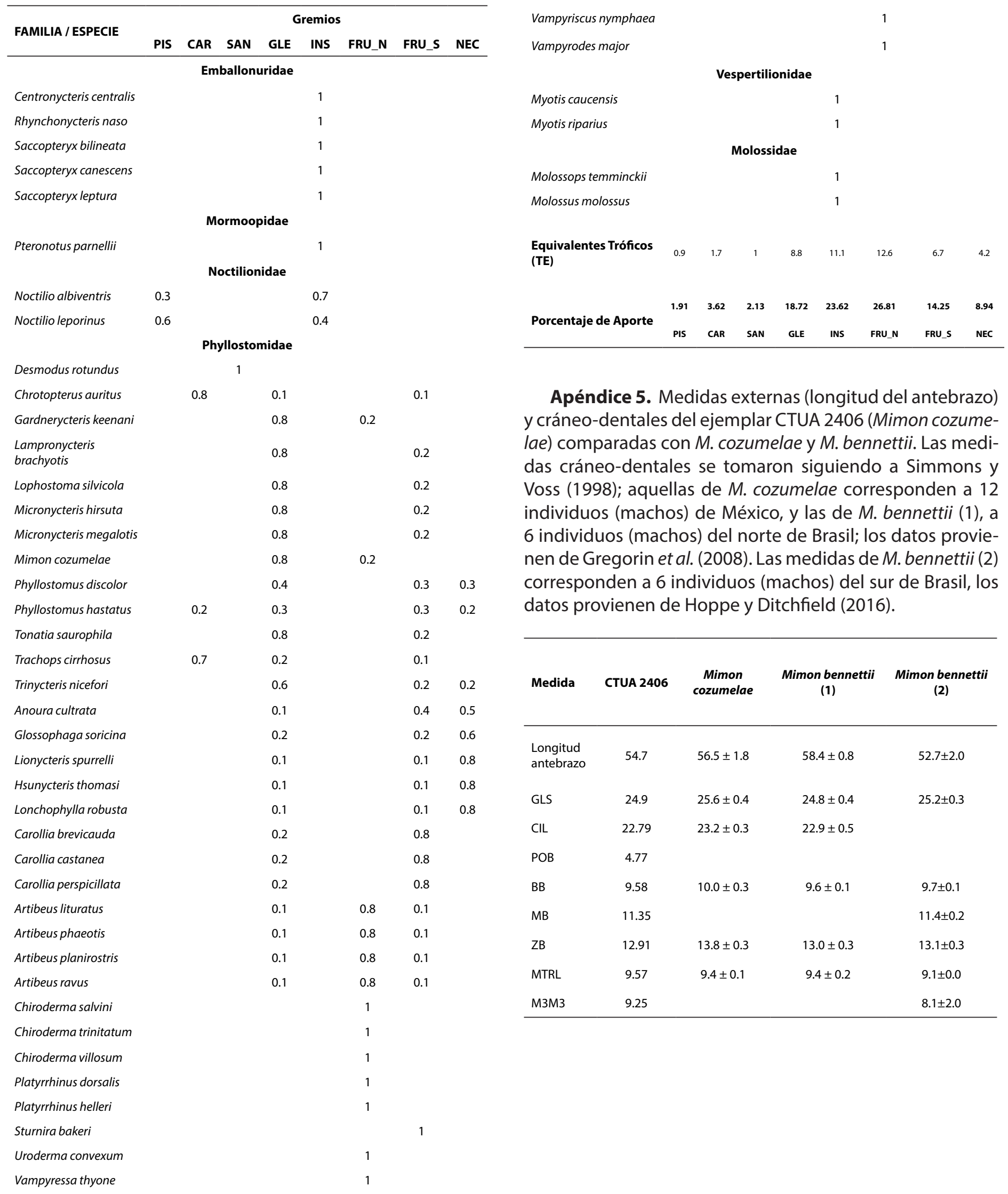


Apéndice 6. Registros correspondientes a especies características de la región. Primera fila (de izquierda a derecha): Centronycteris centralis, Rhynchonycteris naso, Saccopteryx canescens, Pteronotus parnellii; segunda fila: Noctilio leporinus, Desmodus rotundus, Chrotopterus auritus, Gardnerycteris keenani; tercera fila: Lophostoma silvicola, Micronycteris megalotis, Phyllostomus hastatus, Tonatia saurophila; cuarta fila: Trinycteris nicefori, Anoura cultrata, Glossophaga soricina, Lonchophylla robusta; quinta fila: Carollia castanea, Artibeus lituratus, Chiroderma villosum, Sturnira bakeri; sexta fila: Vampyriscus nymphaea, Vampyrodes major, Molossus molossus, Myotis caucensis.

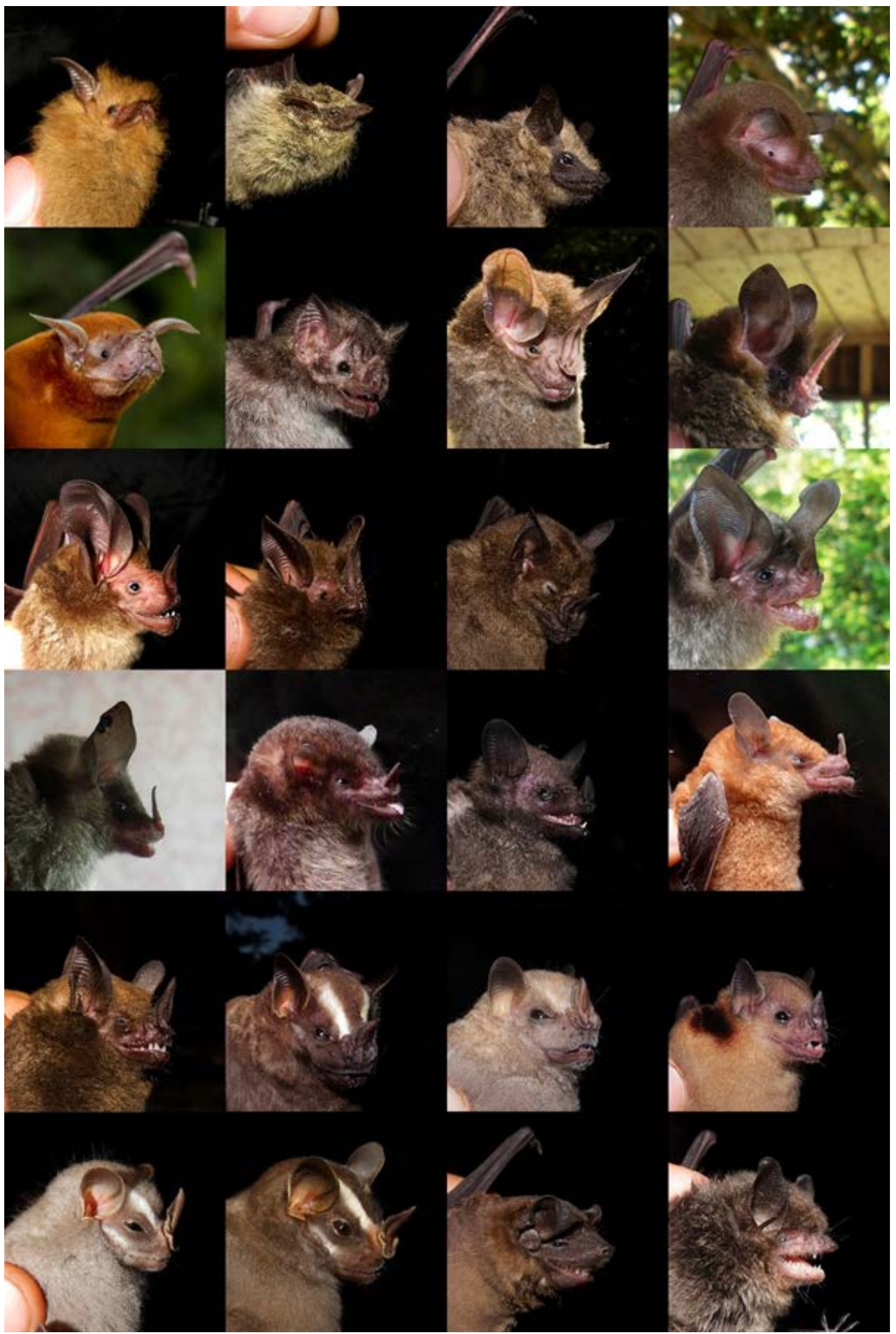

